\title{
A case of Wilms tumor with right ventricular extension of tumor thrombus
}

\author{
Mustafa Çakan*1, Ayșe Gülnur Tokuç ${ }^{1}$, Kıvılcım Karadeniz Cerit ${ }^{2}$, Koray Ak ${ }^{3}$, Rabia Ergelen ${ }^{4}$ \\ ${ }^{1}$ Department of Pediatric Hematology and Oncology, Faculty of Medicine, Marmara University, Istanbul, Turkey \\ ${ }^{2}$ Department of Pediatric Surgery, Faculty of Medicine, Marmara University, Istanbul, Turkey \\ ${ }^{3}$ Department of Cardiovascular Surgery, Faculty of Medicine, Marmara University, Istanbul, Turkey \\ ${ }^{4}$ Department of Radiology, Faculty of Medicine, Marmara University, Istanbul, Turkey
}

Received: November 26, 2016

DOI: $10.5430 /$ crim.v4n1p60
Accepted: December 21, $2016 \quad$ Online Published: January 18, 2017

URL: https://doi.org/10.5430/crim.v4n1p60

\begin{abstract}
Primary renal tumors comprise $6 \%$ of all childhood cancers. Wilms tumor is the most common primary renal tumor in pediatric age group and the peak age of diagnosis is 3-4 years. In $10 \%$ of cases tumor extension into hepatic vein and inferior vena cava can be seen. But tumor extension into whole inferior vena cava, right atrium and right ventricle is only seen in less than $1 \%$ of patients. A 2-year-old girl was admitted to the hospital because of abdominal distension that was noticed by the parents two weeks ago. Imaging studies revealed that she had a mass at the right renal lodge which was favoring to Wilms tumor and on thorax tomography tumor thrombus was seen in the whole inferior vena cava, right atrium and right ventricle. Neoadjuvant chemotherapy was given for 7 weeks. On the 8th week of diagnosis, under cardiopulmonary bypass, surgical operation by pediatric and cardiovascular surgery teams for primary renal tumor and for cavo-atrial tumor thrombus was performed. Pathological examination of the mass was reported as stage 3 diffuse anaplastic Wilms tumor. The patient completed 24 weeks of chemotherapy protocol and she is being followed for 15 months without any morbidity. We present our case to emphasize the importance of multidisciplinary approach in Wilms tumor with cardiac extension.
\end{abstract}

Key Words: Neodjuvant chemotherapy, Tumor thrombus, Wilms tumor

\section{INTRODUCTION}

Wilms tumor constitutes 5\%-6\% of all childhood cancers and most commonly encountered between the ages of 3-4 years. ${ }^{[1]}$ In $5 \%$ of cases the tumor is bilateral. Congenital anomalies occur in $12 \%-15 \%$ of cases. Genitourinary anomalies, aniridia and hemihypertrophy are the most common accompanying anomalies. With surgery and chemotherapy cure rate is around $90 \% .^{[1,2]}$ In less than $10 \%$ of cases, as an extension of tumor tissue, tumor thrombus can be seen at the level of hepatic vein and inferior vena cava (IVC) ${ }^{[3]}$ But extension of thrombus into whole IVC, right atrium and right ventricule (cavo-atrial thrombus) is observed in less than $1 \%$ of patients. ${ }^{[1,3]}$ Since radiographic evaluations are very diagnostic and incisional or tru-cut biopsy can cause seeding metastases, in cases without distant metastases or that did not invade renal sinus, the primary method of treatment is surgery. ${ }^{[1]}$ If tumor thrombus is detected above the level of hepatic vein at the time of diagnosis, to achieve regression, the use of neoadjuvant chemotherapy for at least 5 weeks is recommended. ${ }^{[1,4]}$ In cases with intracardiac extension of tu-

* Correspondence: Mustafa Çakan; Email: mustafacakan@ hotmail.com; Address: Department of Pediatric Hematology and Oncology, Faculty of Medicine, Marmara University, Mimar Sinan Caddesi No.41, Pendik, Istanbul 34899, Turkey. 
mor thrombus, the preferred surgical method is total excision of primary mass and intracardiac thrombus simultaneously under cardiopulmonary bypass. ${ }^{[5-7]}$

\section{Case presentation}

A 2-year-old girl was admitted to the hospital with the complaint of abdominal distension for 2 weeks. On physical examination, a solid mass was palpable on the right part of the abdomen. Hemihypertrophy, aniridia and genitourinary anomalies were not detected. On abdominal computerized tomography (CT), a $63 \mathrm{~mm} \times 72 \mathrm{~mm}$ sized mass with patchy contrast enhancement that was compatible with Wilms tumor was observed at the right renal lodge (see Figure 1). On thorax CT, there were no metastatic lesions in the lung parenchyma but tumor thrombus was observed in the lumen of the hepatic vein, IVC, right atrium and right ventricle (see Figure 2).

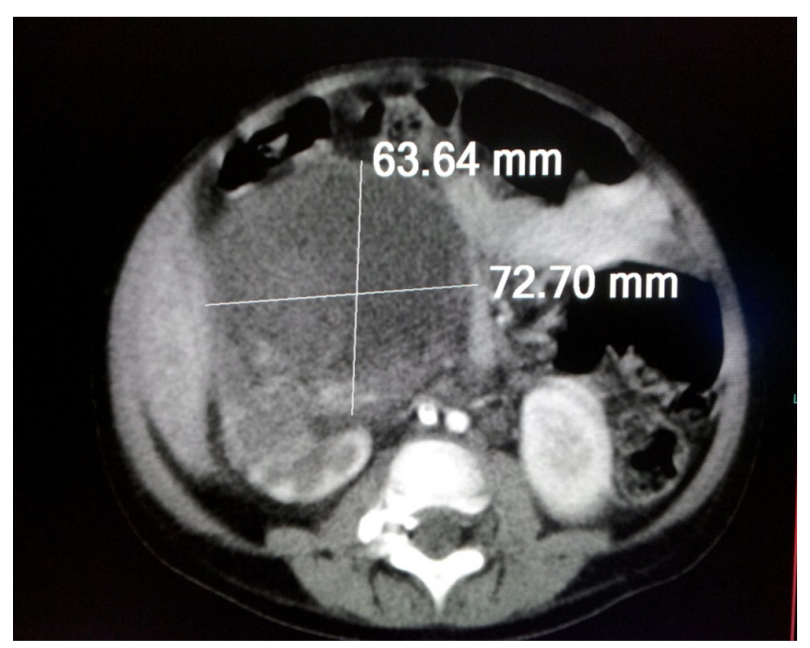

Figure 1. The view of the primary renal mass on the abdominal CT

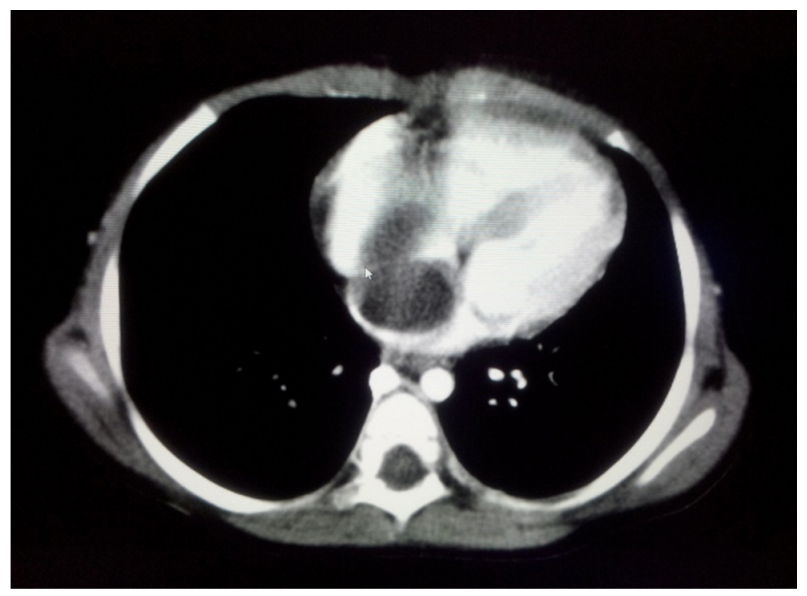

Figure 2. The view of the intracardiac tumor thrombus mass on thorax CT
Neoadjuvant chemotherapy composed of actinomysin-D (45 mcg/ $/ \mathrm{kg})$, doxorubicin $(1.5 \mathrm{mg} / \mathrm{kg})$ and vincristine $(0.05 \mathrm{mg} / \mathrm{kg})$ was started, with the diagnosis of Wilms tumor, to achieve regression of tumor thrombus. On echocardiographic examination, a mass filling whole IVC and right atrium that was extending into right ventricle in each systole was observed. Systolic and diastolic functions of the heart were normal. After consultation with cardiovascular surgery (CVS) department enoxaparin was started to prevent adhesion of the mass to the vascular wall. On the 4th week of neoadjuvant chemotherapy, neither regression of cavo-atrial thrombus nor shrinkage of primary renal mass was observed on imaging studies. On the 8th week of admission, patient was taken into operation by pediatric surgery and CVS teams. At the beginning of the surgery, deep hypothermia and cardiopulmonary bypass was applied by cannulation of IVC, superior vena cava and ascending aorta. Renal mass was reached with abdominal incision. After ligation of renal artery, thrombus in the right atrium was extracted by atrial incision. Balloon catheter was inserted into IVC and thrombus located in the IVC was taken out. With distal caval incision more thrombus was extracted from distal IVC. After ligation of renal vein, primary renal mass was extracted totally (see Figure 3). No complication was observed during or after surgery.

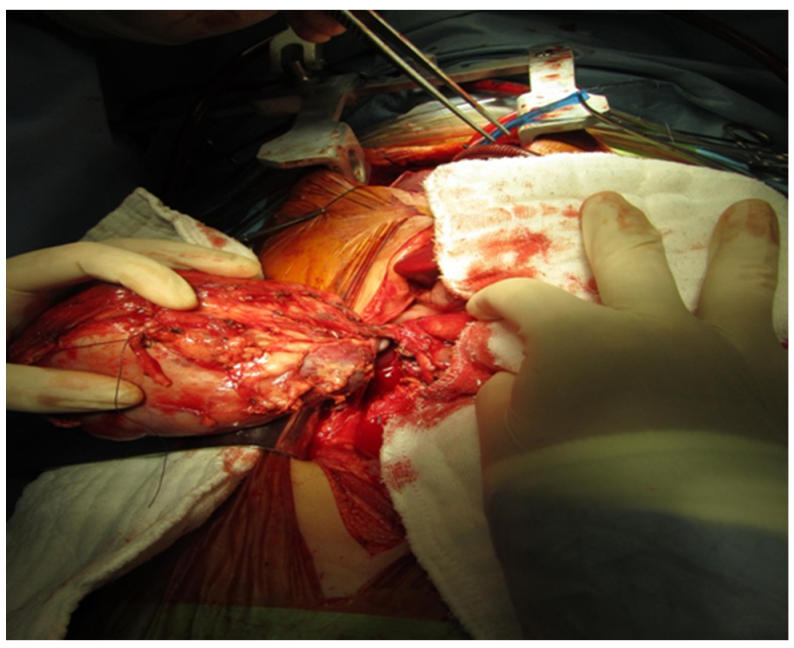

Figure 3. The macroscopic view of the primary renal mass during surgery

Pathological examination of the mass was reported as stage 3 diffuse anaplastic Wilms tumor. On postoperative echocardiographic and tomographic examinations no residual mass or thrombus was detected. Since diffuse anaplasia was seen on pathological examination cyclophosphamide and etoposide were added to chemotherapy regimen and $1080 \mathrm{cGy}$ radiotherapy was applied to the tumor bed. The patient com- 
pleted 24 weeks of chemotherapy and is being followed for 15 months without any morbidity.

\section{Discussion}

Wilms tumor is the most common primary renal tumor in pediatric age group and is the second most common retroperitoneal tumor after neuroblastoma. Ninety percent of cases are diagnosed between the ages of 1-5. ${ }^{[1,8]}$ Most of the cases are sporadic and in $5 \%$ of the cases the tumor is bilateral. The most common presenting symptoms are abdominal mass, distension or hematuria. ${ }^{[1,8,9]}$ Congenital anomalies like aniridia, genitourinary anomalies, hemihypertrophia are seen in $12 \%-15 \%$ of cases. In syndromes like WAGR, DenysDrash and Beckwith-Wiedemann, the risk of Wilms tumor increases. ${ }^{[1,8]}$ There were no accompanying anomalies or dysmorphic features in our case.

In $10 \%$ of cases, the tumor tissue may invade into renal sinus and hepatic vein. In less than $1 \%$ of cases tumor thrombus may fill whole IVC and may extend into right side of the heart. ${ }^{[3,4]}$ Abdullah et al. reported that in 264 Wilms tumor patients 9 of them had cavo-atrial thrombus and 2 of them had right ventricular involvement. ${ }^{[5]}$ In the report of Hadley et al. in 10 of 406 (2.4\%) Wilms tumor patients, extension of thrombus mass into the right atrium and ventricle was observed. ${ }^{[6]}$ At the time of diagnosis, if there is tumor thrombus above the level of hepatic vein, neo-adjuvant chemotherapy for 5-7 weeks and re-evaluation is recommended. ${ }^{[1-3,9,10]}$ This also gives a time for the treatment of accompanying diseases and complications and stabilization of the patient before the major surgery. As our patient had right ventricular thrombus, 7 weeks of neoadjuvant chemotherapy was given. On control evaluation, possibly due to having anaplastic histology, neither shrinkage of the renal mass nor the regression of the cavo-atrial thrombus was observed. In the study of Murthi et al. neoadjuvant chemotherapy was given to 11 out of 13 cases with intravascular extension. However shrinkage of the thrombus did not occur in $30 \%$ of patients just like our case. ${ }^{[7]}$
In cases of Wilms tumor with cavo-atrial thrombus, the recommended surgical method is extraction of the primary renal and thrombus masses simultaneously in one session under cardiopulmonary bypass. ${ }^{[3-7]}$ The overall prognosis of these patients with cavo-atrial thrombus is the same with the same histologic subtype without cavo-atrial thrombus. But as the surgical method is much more complicated, surgical morbidity and mortality is higher in patients with cavo-atrial thrombus. ${ }^{[2]}$ Abdullah et al. reported one mortality due to Budd-Chiari syndrome in 6 patients receiving cardiopulmonary bypass. ${ }^{[5]}$ In the study of Hadley et al. 1 of 7 patients with cavo-atrial thrombus died in the early postoperative period. ${ }^{[6]}$ Murthi et al. did not report any surgical mortality in 8 cases with intracardiac thrombus. ${ }^{[7]}$ Following surgical resection, patients should continue on chemotherapy regimen. In patients with cavo-atrial thrombus who received neoadjuvant chemotherapy, the primary tumor should be considered stage III, regardless of findings at surgery, and post-operative radiation therapy should be given to all such patients. ${ }^{[1,2]}$ Also patients with any residual mass or tumor thrombus are considered stage III and treated accordingly. ${ }^{[1]}$ If the tumor remains inoperable after neoadjuvant chemotherapy and/or radiotherapy, then a biopsy of both the primary tumor and accessible metastatic lesions should be performed. These patients have a very poor prognosis and will require a different chemotherapy regimen. ${ }^{[1]}$ Our case had abdominal, atrial and caval incisions under cardiopulmonary bypass and we did not observe any morbidity during or after surgical procedure. She completed chemotherapy and radiotherapy regimens and is being followed for 15 months without any sign of disease activity.

In conclusion, the possibility of cavo-atrial thrombus should be kept in mind in patients with Wilms tumor. In patients with intracardiac extension, multidisciplinary approach is indispensable to reduce the morbidity and mortality.

\section{CONFlicts OF InTEREST Disclosure}

The authors have no competing interests to declare.

\section{REFERENCES}

[1] Gratias E. Renal Tumors. In Lanzkowsky P (ed). Manual of Pediatric Hematology and Oncology. 5th edition. San Diego, CA: Elsevier. 2011: 695-714.

[2] Kalapurakal JA, Dome JS, Perlman EJ, et al. Management of Wilms tumour: Current practice and future goals. Lancet Oncol. 2004; 5: 3746. PMid: 14700607 http://dx.doi.org/10.1016/S1470-204 5 (03) 01322-6

[3] Cristofani LM, Duarte RJ, Almedia MT, et al. Intracaval and intracardiac extension of Wilms tumor. The influence of preoperative chemotherapy on surgical mortality. Int Braz J Urol. 2007; 33: 683-9. PMid: 17980066 https ://doi.org/10.1590/S1677-5538200 7000500010

[4] Ritchey ML, Kelalis PP, Haase GM, et al. Preoperative therapy for intracaval and atrial extension of Wilms tumor. Cancer. 1993; 71: 4104-4110. PMid: 8389661 https : //doi .org/10.1002/1097-0142(19930615)71: 12<4104: : AID-CNCR2820711249>3.0.CO;2-7

[5] Abdullah Y, Karpelowsky J, Davidson A, et al. Management of nine cases of Wilms tumour with intracardiac extension - a single centre 
experience. J Pediatr Surg. 2013; 48(2): 394-9. PMid: 23414872 https://doi.org/10.1016/j.jpedsurg. 2012.11.024

[6] Hadley GP, Sheik-Gafoor MH, Buckels NJ. The management of nephroblastoma with cavo-atrial disease at presentation: experience from a developing country. Pediatr Surg Int. 2010; 26(12): 116972. PMid: 20697900 https ://doi.org/10.1007/s00383-010 $-2667-5$

[7] Murthi GV, Koçyıldırım E, Sellathury S, et al. Wilms tumour with persistent intravascular extension: A review of the surgical aspects of management. J Pediatr Urol. 2006; 2: 439-45. PMid:18947653 https://doi.org/10.1016/j.jpurol.2005.10.004
[8] Birch JM, Breslow N. Epidemiologic features of Wilms tumor. Hematol Oncol Clin North Am. 1995; 9: 1157-78. PMid:8591959

[9] Dome JS, Roberts CM, Argani P. In: Orkin SH, Ginsburg D, Nathan DG, et al., eds. Oncology of Infancy and Childhood. Elsevier Academic Publishers. 2009: 541-73. https://doi .org/10.1016/B9 78-1-4160-3431-5.00015-7

[10] Akyüz C, Yalçin B, Yildiz I, et al. Treatment of Wilms tumor: a report from the Turkish Pediatric Oncology Group (TPOG). Pediatr Hematol Oncol. 2010; 27(3): 161-78. PMid:20367260 https: //doi.org/10.3109/08880010903447375 